\title{
La lucha por ser indígenas en la ciudad: El caso de la comunidad shipibo-konibo de Cantagallo en Lima ${ }^{1}$
}

\section{The struggle to be indigenous in the city: The case of the shipibo-konibo community of Cantagallo in Lima}

\author{
Oscar Espinosa ${ }^{2}$ \\ Pontificia Universidad Católica del Perú
}

\section{Resumen}

El número de indígenas que en América Latina viven y reproducen su cultura en las grandes ciudades ha ido creciendo

1 Quiero agradecer a la comunidad shipiba de Cantagallo, así como a sus líderes, especialmente Juan Agustín, Ricardo Franco y Karina Pacaya. También quiero agradecer a mis alumnas y alumnos de la PUCP con quienes hemos conversado sobre estos temas a lo largo de varios años. De manera particular quiero mencionar a Juan Carlos Zegarra, José Carlos Ortega, Guillermo Peláez, Javier Lazarte, Arturo Gutiérrez, y a los miembros del Grupo Interdisciplinario de Amazonía (GIA) de la PUCP que participaron en el proyecto "Barrio Shipibo" así como a Kukuli Tenorio, Diana Álvarez, Isabel Urrutia, Richard O’Diana y otros amigos con quienes hemos apoyado en distintos momentos las demandas de la comunidad de Cantagallo frente a la Municipalidad de Lima Metropolitana.

2 Doctor en Antropología, profesor principal del Departamento de Ciencias Sociales de la Pontificia Universidad Católica del Perú.

Correo electrónico: oespinosa@pucp.edu.pe 
en las últimas décadas. Los pueblos indígenas de la Amazonía peruana no son ajenos a esta dinámica, y existen algunos, como el pueblo shipibo-konibo que ha venido creando comunidades urbanas que les permiten reproducir su propia cultura en un nuevo contexto espacial. Este proceso se da, además, en un contexto difícil, en la medida en que el Estado peruano no reconoce oficialmente la existencia de comunidades indígenas urbanas. Esta situación ha generado un proceso de movilización y lucha por el derecho a vivir como indígenas en la ciudad. El presente artículo busca discutir cómo la comunidad shipiba de Cantagallo ha venido luchando por la posibilidad de vivir como indígenas en la ciudad de Lima.

Palabras claves: shipibo-konibo, política indígena, indígenas urbanos, derechos indígenas, movimiento indígena, Amazonía, Lima..

\section{Abstract}

The number of indigenous people living in Latin America and reproducing their culture in large cities has been growing in recent decades. The indigenous peoples of the Peruvian Amazon are no strangers to this dynamic, and there are some, such as the Shipibo-Konibo people that have been creating urban communities that allow them to reproduce their

154 own culture in a new spatial context. This process is also taking place in a difficult context, insofar as the Peruvian State does not officially recognize the existence of urban indigenous communities. This situation has generated a process of mobilization and struggle for the right to live as indigenous people in the city. This article discuss how the Shipibo community of Cantagallo has been fighting for the possibility of living as indigenous people in the city of Lima. 
Key words: shipibo-konibo, indigenous politics, urban indigenous, indigenous rights, indigenous movement, Amazon, Lima...

\section{Introducción}

En las últimas décadas la población indígena amazónica que vive en ciudades ha ido creciendo significativamente. La antropología también ha comenzado a mostrar interés por este fenómeno, como se puede apreciar en el creciente número de investigaciones, tesis y publicaciones dedicadas a ello. ${ }^{3}$ Sin embargo, no todas las sociedades indígenas de la Amazonía se relacionan de la misma manera con lo urbano. En algunos casos se prefiere urbanizar las propias comunidades indígenas, mientras que, en otros, la opción es la de migrar hacia las grandes ciudades.

En el caso de la Amazonía peruana, la migración se orienta principalmente hacia las ciudades que se ubican en la misma región, ya que permite a los migrantes no alejarse tanto del mundo social y natural del que provienen. La migración a otras ciudades peruanas no amazónicas ha sido menor, aunque aparentemente la migración hacia Lima se vendría incrementando en los últimos años debido a la concentración

3 Existe una creciente bibliografía sobre distintos aspectos relacionados a la situación de los indígenas urbanos amazónicos, principalmente en Brasil, Colombia y Perú. Entre ésta, cabe resaltar el número especial dedicado al tema del Journal of Latin American and Caribbean Studies editado por Miguel Alexiades y Daniel Peluso (2015). Para una visión panorámica sobre el caso de los indígenas amazónicos en Lima cabe resaltar los trabajos de Oscar Espinosa (2009), Ismael Vega (2014) y Peñaranda (2015). 
de servicios y de ofertas de empleo, por lo menos de parte de los pueblos indígenas que viven en la región amazónica más cercana, como es el caso de los pueblos asháninka, yánesha y shipibo, y que se conectan con esta ciudad a través de la llamada "carretera central".

Es difícil saber con exactitud cuántos indígenas amazónicos viven en Lima o en otras ciudades peruanas actualmente debido a la ausencia de una adecuada información censal. Los censos de población indígena del Instituto Nacional de Estadística e Informática (INEI) disponibles (realizados en 1993 y 2007), ${ }^{4}$ sólo tomaron en cuenta a la población indígena viviendo en comunidades rurales y no a los que viven en las ciudades. Por ello, es probable que la población indígena que vive en Lima $^{5}$ o en otras ciudades del país pudiera ser estadísticamente más relevante.

Una vez en la ciudad, los migrantes indígenas amazónicos se insertan de manera distinta en este nuevo espacio geográfico,

4 El último Censo Nacional de Población de 2017 incluyó una pregunta sobre auto-identificación étnica, sin embargo, los resultados censales todavía no están disponibles a agosto de 2018.

5 Según el Censo Nacional de Población de 2007, el número de personas residente en la ciudad de Lima que reconoce tener como lengua materna una lengua indígena amazónica fue de 6400. Sin embargo, este indicador -la lengua materna como criterio exclusivo de identidad étnica- resulta problemático, más aún en los contextos urbanos, donde muchas personas indígenas evitan su uso - al igual que otras formas de manifestaciones étnicas - para evitar ser discriminados. Por ello el número de migrantes indígenas es mucho mayor al reconocido. Así, por ejemplo, por información que circula entre los propios indígenas y sus redes sociales se sabe de la existencia de redes de migrantes asháninka y yánesha que viven y trabajan en la ciudad de Lima, pero que no aparecen registrados en ningún censo. 
social y cultural. En muchos casos, estos migrantes buscan pasar desapercibidos, eliminando cualquier signo de expresión de la etnicidad, con el fin de evitar la discriminación por parte de sus vecinos o posibles empleadores, o limitando estas expresiones al círculo de los parientes o de personas más cercanas. Sin embargo, migrantes de otros pueblos como los del pueblo shipibo-konibo no tienen problema por expresar su identidad étnica, y hasta buscan espacios propicios para hacerlo, incluso en contextos como el de la ciudad de Pucallpa donde siguen siendo discriminados. ${ }^{6}$

Así, por ejemplo, se visibilizan a través de la presencia de las mujeres vestidas con atuendos tradicionales que venden sus artesanías de manera ambulatoria, pero también se hacen presentes a través de los medios de comunicación y en diversos actos públicos en las instituciones públicas y en las calles de la ciudad. En este proceso, los diseños geométricos o kené que las mujeres shipibas pintan o bordan en su cerámica y sus telas se pueden encontrar reproducidos en otros medios: en las paredes, avisos publicitarios o ropa de moda, incluyendo minifaldas, bikinis y bolsos o carteras.

Pero además de esta visibilidad, en diversas ciudades peruanas, las familias shipibo-konibo (o shipibo simplemente, como las llamaré de ahora en adelante), han optado por configurar lo que ellos mismos denominan "comunidades interculturales" donde no solamente viven en viviendas contiguas, sino donde también reproducen o adaptan formas de organización social y política, y buscan ser reconocidas como comunidades indígenas en medio de la ciudad. Esta lucha es

6 Al respecto resulta interesante el caso estudiado por Sara Sarfaty (2011) en relación a los estudiantes shipibos en la ciudad de Pucallpa. 
más crítica en la medida en que el estado peruano no reconoce formalmente la existencia de comunidades indígenas urbanas.

\section{Las comunidades indígenas ¿̨en la ciudad?}

Según la legislación peruana solamente existe la figura jurídica de la "comunidad nativa" que corresponde a las comunidades rurales, tal como lo establece la "Ley de Comunidades Nativas". ${ }^{7}$ Esta ha sido la forma en que el Estado peruano decidió, a inicios de la década de 1970, organizar el espacio de las sociedades amazónicas en lugar de reconocer territorios más amplios como ocurre en países vecinos. Las razones detrás de esta decisión fueron múltiples y complejas, y aparentemente correspondieron a una combinación de modelos, principalmente de la comunidad campesina andina, así como de las cooperativas de producción y comercialización, entre otros.

La reducción del espacio territorial al más pequeño de la comunidad local no solamente ignora la concepción indígena del territorio y su uso real del espacio, sino permite dejar espacios libres para promover la colonización y la explotación de recursos naturales por población no indígena. Por ello, la figura jurídica de la "comunidad" como forma de organización territorial indígena se viene cuestionando desde que

7 En 1974, el gobierno militar del general Velasco Alvarado promulgó el Decreto Ley 20653 (Ley de Comunidades Nativas y de Promoción Agropecuaria de las Regiones de Selva y Ceja de Selva). En 1978, el gobierno del general Morales Bermúdez derogó esta ley, y fue reemplazada por la Ley 22175 (Ley de Comunidades Nativas y de Desarrollo Agrario de la Selva y Ceja de Selva). Esta ley, conocida como "Ley de Comunidades Nativas" es la actualmente vigente. 
se creó en el año 1974, y de ahí que este proceso haya sido calificado como "despojo institucionalizado" por connotados antropólogos y abogados vinculados al movimiento indígena peruano (Barclay y Santos 1980, Chirif 1980, Santos \& Barclay 1985, Chirif, García Hierro y Smith 1991, Chirif y García Hierro 2007). Este despojo se acentuó a partir de 1978, cuando el gobierno del General Morales Bermúdez promulgó una segunda "Ley de Comunidades Nativas", la Ley No. 22175, que derogó la de 1974. Esta nueva ley, titulada "Ley de Comunidades Nativas y de Desarrollo Agrario de la Selva y Ceja de Selva”, eliminó la integridad territorial de las comunidades nativas, al declarar que las tierras de aptitud forestal no son de propiedad de la comunidad indígena, sino que son cedidas en uso por parte del Estado (art.11). Esta ley es la actualmente vigente, y sigue cuestionada tanto por las organizaciones indígenas como por los especialistas. Sin embargo, a pesar de sus limitaciones, constituye la única protección legal para los territorios indígenas amazónicos (Chirif y García Hierro 2007), aunque esta aceptación pragmática no implica claudicar en la demanda al Estado del reconocimiento legal de sus territorios integrales o ancestrales.

Pero además de los impactos sobre el territorio y el vínculo con el espacio y la naturaleza, la creación de las comunidades nativas también generó nuevas formas de organización social, política y económica para los indígenas. Se ha criticado, en este sentido, que la forma jurídica de la "comunidad nativa" no corresponde a la forma tradicional de organización social y política, y, por lo tanto, termina siendo una medida impuesta para "modernizar" las sociedades indígenas e incorporarlas al mercado sin tomar en consideración los intereses o beneficios para los propios indígenas (Ludescher 1986, Espinosa 2004). 
La figura de la "comunidad nativa" implica, además del reconocimiento por parte del Estado de tierras donde pueden vivir las familias indígenas, ${ }^{8}$ el establecimiento de nuevas formas de organización social y política que incluyen, por ejemplo, la figura de un jefe de la comunidad (que en algunas regiones es llamado "presidente" o "apu"), de una junta directiva, etc. En otras palabras, se podría decir que una "comunidad nativa" es al mismo tiempo una forma de configuración del espacio local y una serie de relaciones sociales y políticas basadas en relaciones de parentesco.

Hoy en día, a pesar de todos estos impactos, el modelo de la "comunidad nativa" ha sido en buena parte apropiado por las sociedades amazónicas peruanas, sin llegar a desaparecer totalmente otras formas más tradicionales de organización espacial, social y política (Rosengren 1987, Narby 1989). En este sentido, se podría decir que la idea de "comunidad" coexiste con otras formas indígenas de entender el territorio, el parentesco o el liderazgo político. En este sentido, las "comunidades interculturales urbanas" del pueblo Shipibo reproducen estas dos características. Es decir, no se trata solamente de un grupo de vecinos que viven cerca unos de otros y que comparten ciertos rasgos culturales y étnicos; sino de una auténtica comunidad que comparte el mismo espacio geográfi-

8 Es importante señalar que la Ley de Comunidades Nativas vigente no reconoce la "propiedad" sobre el espacio demarcado por el Estado para las comunidades. Los indígenas solamente son dueños de las tierras dedicadas a la vivienda, la agricultura y la ganadería. Los bosques están cedidos en uso, y los cuerpos de agua como lagunas, cochas o ríos tampoco son incluidos como propiedad comunal, a pesar de que el bosque y el agua son espacios fundamentales en la definición del territorio para las sociedades indígenas amazónicas (Surrallés y García Hierro 2004). 
co, que está organizada políticamente como unidad, y cuyas familias comparten en gran medida lazos de parentesco.

Vivir en "comunidad" significa vivir juntos y reproducir en la ciudad, de la mejor manera posible, ciertos aspectos de su vida tal como la realizan en sus comunidades de origen ubicadas en las riberas del río Ucayali y sus afluentes. Fundamentalmente buscan reproducir la forma de organizar el espacio, sus viviendas y su vida cotidiana. Pero además, en el caso de las comunidades urbanas shipibas encontramos que el uso del término "comunidad" está también asociado a una demanda de reconocimiento como "comunidades nativa”, en un contexto en que la ley no contempla la existencia de comunidades indígenas urbanas, a diferencia de Colombia, por ejemplo, que hoy en día reconoce los cabildos indígenas en las ciudades (Del Cairo y Rozo 2006, Bonilla 2011, Sánchez 2012).

\section{La comunidad shipiba de Cantagallo en Lima}

En la ciudad de Lima, un grupo importante de familias shipibas que migraron en distintas épocas desde la región Ucayali se asentaron en el barrio de Cantagallo, en un lugar relativamente cercano al centro histórico de Lima, y ubicado a las orillas del río Rímac que atraviesa la ciudad. Este asentamiento comenzó a crecer sobre todo después del año 2000.

El número de migrantes shipibos que comenzaron a quedarse a vivir en Lima de forma más permanente comenzó a crecer desde el año 1991, pero vivían dispersos en diferentes partes de la ciudad. Anteriormente, la mayoría de shipibos viviendo en Lima lo hacían de manera temporal: como dirigentes o 
como estudiantes. Ya desde entonces también crearon una primera asociación de artesanas en la ciudad: la Asociación Interétnica para el Desarrollo de la Artesanía en la Amazonía Peruana (AIDAAP), pero no llegó a prosperar, como veremos más adelante.

La comunidad shipiba de Cantagallo recién se forma hacia el año 2000. La historia mítica de la comunidad establece sus orígenes en la llamada "Marcha de los Cuatro Suyos" organizada por el entonces candidato presidencial Alejandro Toledo en contra del gobierno corrupto del presidente Fujimori. Según relatan algunos miembros de la comunidad, cerca de 30 shipibos que participaron activamente en esta marcha de protesta que no tenían el dinero para volver a Ucayali que les habían ofrecido, deciden quedarse a vivir en Lima.

Otra versión, posiblemente más cercana a lo que ocurrió realmente, señala que algunos de los shipibos que vivían en Lima vendiendo artesanía fueron contactados por los organizadores de una feria artesanal que se iba a realizar en un mercado ubicado en el barrio de Cantagallo. Al finalizar la feria, siete familias pidieron permiso para quedarse a vivir en el segundo nivel del mercado (Zegarra 2009: 33-34). Unos meses después, el número de familias se había mul162 tiplicado a catorce $(\mathrm{p}, 35)$. Desde entonces, más familias

9 Esta versión es la preferida para contar a visitantes y a los medios de comunicación, debido al carácter épico de la historia. Sin embargo, se trata de una versión parcialmente real, en la medida en que nunca la falta de apoyo económico ha sido un impedimento para viajar, ya que se pueden conseguir los fondos con trabajos temporales, venta de artesanía o préstamos de familiares. 
shipibas han seguido llegando a este lugar hasta llegar a ser más de doscientas sesenta familias en total. ${ }^{10}$

El barrio de Cantagallo no es una zona espacialmente homogénea, sino que está compuesta por tres sectores o "niveles", ya que están ubicados en alturas diferentes. ${ }^{11} \mathrm{El}$ primer nivel corresponde al Mercado "Las Malvinas" donde se ubican quioscos dedicados a la venta de artículos y herramientas de ferretería, además de puestos de comida y bodegas. En el segundo nivel se ubican diversas familias "mestizas" y shipibas. En esta zona se encuentra la losa deportiva, los locales o templos de las iglesias evangélicas, además de bodegas y puestos de comida. En este nivel también está ubicada la escuela. Finalmente, el tercer nivel ${ }^{12}$ está poblado mayoritariamente por familias shipibas, y es donde viven la mayoría de artistas y artesanos. ${ }^{13}$

10 En el proceso de negociación con la Municipalidad Metropolitana de Lima para su reubicación, han sido oficialmente aceptadas 265 familias empadronadas.

11 Esta descripción está hecha usando el indicativo presente, pero el barrio ha sido totalmente modificado a partir del año 2014 para la construcción de la autopista, por lo que se debería haber escrito en tiempo pasado. El mismo comentario se aplica al plano que se presenta a continuación.

12 El tercer nivel es el más alto y se formó sobre una montaña de desmonte, desperdicios y basura.

13 En Cantagallo existen tres organizaciones indígenas: AVSHIL (Asociación de Vivienda de Shipibos en Lima) que agrupa principalmente a familias que viven en el segundo nivel, mientras que las del tercer nivel están, en su mayoría, asociadas a ASHIREL y más recientemente también a ACUSHIKOLM (Asociación de la Comunidad Urbana Shipibo-Konibo de Lima Metropolitana). 


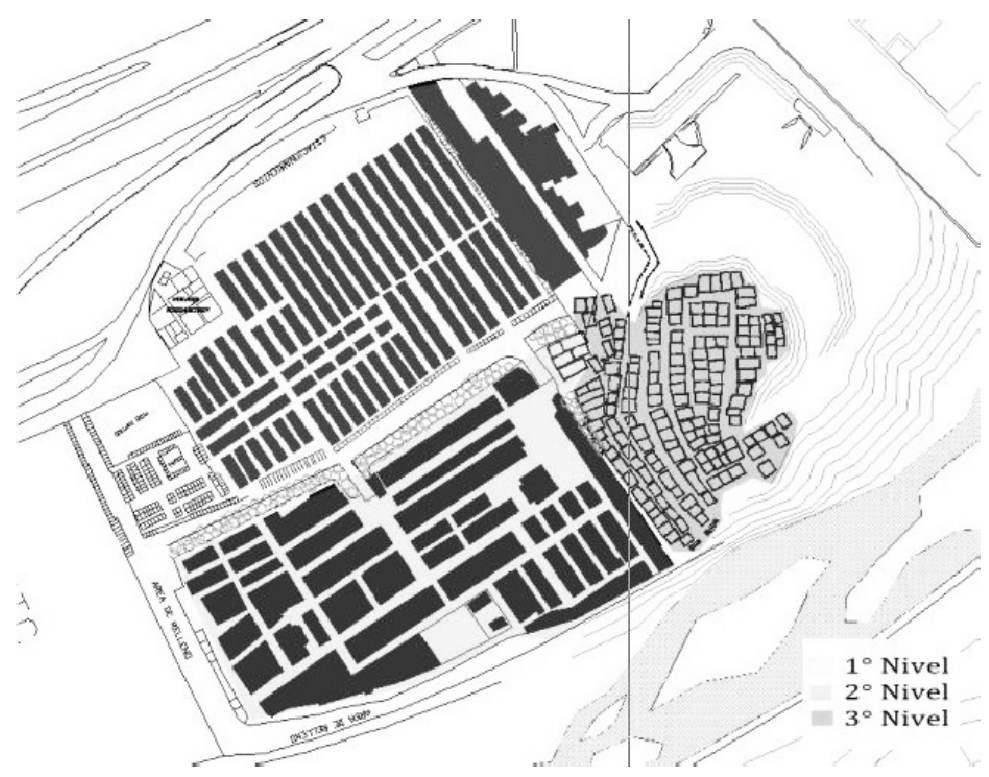

Croquis de la comunidad de Cantagallo en 2014, antes de iniciarse el proceso de reubicación. (Cortesía del Arq. Javier Lazarte).

\section{La lucha por el derecho a vivir como comunidades indí- genas en la ciudad}

La comunidad de Cantagallo no es la única comunidad urbana shipiba. Y al igual que estas otras, está buscando la manera de ser reconocidos oficialmente como comunidad indígena.

164 Pero, además, a diferencia de los otros casos, como el de las comunidades de Tingo María, Pucallpa o San Ramón, el caso de Lima constituye el desafío más grande, ya que se trataría de la primera comunidad indígena amazónica reconocida fuera de la Amazonia. ${ }^{14}$

14 La comunidad shipiba de Cantagallo, además, ha atraído a investigadores de diversas disciplinas en los últimos años. Entre los principales trabajos publicados, 
El caso de Cantagallo ha resultado, pues, el más conflictivo, sobre todo en la medida en que su existencia misma como barrio o comunidad se ha puesto en riesgo a partir del proyecto de una autopista promovido por la Municipalidad de Lima Metropolitana, generando incluso tensiones entre distintas instancias del Estado. Así, por ejemplo, a pesar de no existir una ley que reconozca la existencia de comunidades indígenas urbanas, el Ministerio de Cultura reconoce la existencia de esta comunidad en su Base de Datos de Pueblos Indígenas. ${ }^{15}$

Desde hace ya algunos años, cuando el número de familias shipibas comenzó a crecer y la comunidad de Cantagallo comenzó a consolidarse como tal, se iniciaron distintos procesos para su reconocimiento formal y para asegurar sus viviendas, su escuela y sus espacios comunales. Así, por ejemplo, durante el gobierno de Alan García se inició un primer proceso de diálogo, sin embargo, las autoridades no reconocían a ASHIREL (Asociación de Artesanos Shipibos Residentes en Lima) como interlocutor válido debido a que era una asociación de "artesanos" y no una asociación de "vecinos". Por eso, se cambió el nombre de esta asociación añadiéndole la palabra "pro-vivienda" y una letra más a su sigla para convertirse en la actual ASHIREL-V.

se encuentran los relativos al uso de la lengua shipiba en la comunidad (Zavala y Bariola 2007 y 2008, Bariola 2009, Huaroc 2009, Sánchez et al. 2018), a la producción artística y artesanal (Castillo 2013, Ala 2014), a la educación intercultural bilingüe (Moscoso 2010, Quispe 2011, Anzualdo 2016), a dimensiones identitarias y psicológicas (Ananco 2015, Sánchez 2015, Frigola 2016, Tintaya 2017), a su situación de pobreza (Colán 2009, Arista Zerga 2011, Bariola 2014, Kennedy 2011), a su adaptación, organización y vida general en la ciudad (Anzualdo 2015, Arana 2011, Guima 2011, Lazarte 2011, Strauch et al 2014, Espinosa 2016), entre otros.

15 http://bdpi.cultura.gob.pe/pueblo/shipibo-konibo 
Posteriormente ASHIREL elaboró un proyecto para la creación de un "Centro Turístico-Ecológico Vivencial ShipiboKonibo" (Ashirel-V 2011). Este proyecto incluía el reconocimiento del terreno de la comunidad donde se articulaban espacios para las viviendas de las familias shipibas con espacios para la producción y venta de arte, artesanía, comida, etc. En cierto sentido, el proyecto se parece al de la llamada "Aldea indígena" promovida también por artesanos shipibos en la ciudad de La Merced (Chanchamayo, Junín), aunque esta última es una comunidad mucho más pequeña.

Las negociaciones de ASHIREL y de AVSHIL con las autoridades quedaron truncas cuando la Municipalidad de Lima Metropolitana inicia el proyecto "Línea Amarilla" a fines del año $2009^{16}$ que consiste en la construcción de una autopista que pasaría por el lugar donde está ubicada la comunidad de Cantagallo. Este proyecto conlleva, entonces, la reubicación de las familias que ahí viven. Sin embargo, a diferencia de las familias mestizas que allí viven, las familias shipibas quieren seguir viviendo como comunidad y por ello quieren ser reubicadas juntas. Posteriormente, cuando la alcaldesa Susana Villarán asume la Municipalidad Metropolitana de Lima, el proyecto "Línea Amarilla" se convierte primero en el proyecto "Parque Vía Rímac-Línea Amarilla", al que se le añade luego el proyecto "Río Verde"

166 que busca recuperar las riberas del río Rímac que incluiría también la creación de un parque, el "Parque Cantagallo",

16 El contrato de concesión del Proyecto "Línea Amarilla" entre la Municipalidad Metropolitana de Lima y la empresa que asume el nombre del proyecto, Línea Amarilla S.A.C. (LAMSAC), se firmó el 12 de noviembre de 2009. Esta empresa ad-hoc depende de la empresa brasileña OAS. 
en el lugar donde está ubicada la comunidad shipiba, y por lo tanto, también la reubicación de ésta en un nuevo lugar, y que posteriormente sería denominado el "Proyecto de Vivienda Shipibo-Konibo".

En este contexto, en el mismo año 2011 la Municipalidad Metropolitana de Lima, intentando respetar los derechos indígenas reconocidos por el Convenio 169 de la OIT, inició un proceso de diálogo con la comunidad para negociar el proceso de reubicación. Así, en agosto de 2012, la Municipalidad Metropolitana de Lima organizó un "Taller de Pre-Consulta" ${ }^{17}$ Como resultado de este taller, la comunidad shipiba ha solicitado: un título de propiedad colectivo o comunitario, mantener la escuela intercultural bilingüe, con espacio para una canchita de fútbol o multiusos, ${ }^{18}$ local comunal, lavanderías comunales, y espacios para producción y venta de artesanía (Taller de Pre-consulta. 2012).

Resulta particularmente relevante el pedido de un título colectivo o comunitario. Este acuerdo, sin embargo, no fue automático, sino que supuso diversos debates al interior de la comunidad y de sus organizaciones. Entre los principales argumentos a favor del título comunitario aparecía con claridad el deseo de mantener la comunidad, de evitar su división o su desaparición; así como argumentos relacionados a mantener o reproducir la cultura shipiba. Así, por ejemplo, Luz Fran-

17 Este taller fue dirigido por la Dra. Raquel Yrigoyen, abogada experta internacional en derechos indígenas.

18 La cancha de fútbol constituye un lugar muy importante en configuración espacial de las comunidades indígenas en la Amazonía rural y también en las comunidades urbanas shipibas. La cancha no es sólo un espacio para practicar deporte, sino sobre todo un espacio comunal de encuentro. 
co, importante líder de AVSHIL señalaba que hay diferencias entre la propiedad individual y colectiva: "En la colectiva tenemos que cuidar nuestra cultura, cuidarnos. Tiene que tener una fuerte identificación como comunidad. Nos cuidamos entre todos." (Asamblea del 29 de setiembre de 2013).

Para poder financiar el proyecto "Río Verde" que incluye la construcción del parque, la recuperación de las riberas del río Rímac y la reubicación de la comunidad, la Municipalidad de Lima Metropolitana firma el 15 de febrero de 2013 una adenda al contrato de concesión con la empresa LAMSAC. ${ }^{19}$ Una vez asegurado el dinero, el 16 de mayo de 2013 se firma la Resolución de Alcaldía N 124-2013, por la cual se instala una Mesa de Trabajo compuesta por representantes de la Municipalidad y de las tres organizaciones de la comunidad: ASHIREL-V, AVSHIL y ACUSHIKOLM para discutir diversos temas relacionados al proceso de reubicación de la comunidad, teniendo como punto de partida los acuerdos presentados en el Taller de Pre-consulta de 2012. Esta Mesa de Trabajo se reunió en seis ocasiones durante los ańos 2013 y 2014, llegando a algunos acuerdos importantes. ${ }^{20}$ Entre éstos, el 11 de oc-

19 Bajo este marco legal, el 9 de mayo de 2013 se firma un Contrato de Fideicomiso en Administración, entre LAMSAC (fideicomitente), MML (fideicomisario) y Scotiabank Perú (fiduciario).

20 Estas seis sesiones consistieron en: (1) instalación del Grupo de Trabajo (23.05.2013); (2) definición de la agenda y metodología del Grupo de Trabajo (05.06.2013); (3) informes sobre documentación del terreno propuesto para la reubicación y sobre el padrón de integrantes de la comunidad (03.07.2013); (4) informes sobre avances en el proceso de acreditación y elaboración de expedientes individuales de la población a ser reasentada (25.07.2013); (5) presentación del diseño de viviendas y equipamientos a cargo del Arq. Augusto Ortiz de Zevallos (29.01.2014): 
tubre de 2013 se aprobó y publicó una resolución ${ }^{21}$ que establecía los lineamientos para identificar quiénes iban a ser considerados en el proceso de reubicación como parte de la comunidad shipiba, logrando determinar un total de 226 familias shipibas por reubicarse.

Este proceso de negociación se complicó a partir de mayo de 2014, cuando comenzaron las obras de remoción de tierra para la construcción de la autopista, recortando significativamente el área ocupada por las viviendas de las familias shipibas en Cantagallo. Así, la empresa trasladó la escuela primaria intercultural bilingüe a la canchita de fútbol de la comunidad y en el mismo lugar también se colocaron casas prefabricadas para las familias que vivían en el área recortada. Pero además, las obras de construcción están afectando la salud de la población, no sólo por el ruido y el polvo, sino sobre todo al liberar contaminantes provenientes de la basura, ya que parte de la comunidad está asentada sobre un relleno sanitario.

Finalmente, el 15 de setiembre de 2014, en asamblea comunal, los presidentes de las tres asociaciones shipibas (ASHIREL-V, AVSHIL y ACUSHIKOLM) en representación del conjunto de la comunidad, aceptan que la comunidad sea

(6) informes sobre avances del proceso y sobre problemas generados en las viviendas y en la Escuela a partir de los trabajos realizados en el segundo y tercer nivel de Cantagallo para la construcción del eje vial (24.04.2014). (Agradezco especialmente a Isabel Urrutia por esta información).

21 "Lineamientos para la identificación de la población perteneciente a la Comunidad Urbana Shipibo- Konibo y residentes no shipibos a ser reubicados por encontrarse dentro del área de influencia directa del Proyecto Vía Parque Rímac” (Resolución de Gerencia No 112-2013MML/GPIP). 
reubicada en un terreno conseguido por la Municipalidad de Lima en la urbanización Campoy, en el distrito de San Juan de Lurigancho, ${ }^{22}$ siempre y cuando también se garantice el traslado de la escuela intercultural bilingüe. Para asegurar el proceso de construcción de las viviendas del entonces llamado "Complejo Habitacional Shipibo Konibo" en el contexto del fin del periodo de la alcaldesa Susana Villarán al frente de la Municipalidad de Lima Metropolitana, emitió el Decreto de Alcaldía No 16 el día 22 de diciembre de 2014.

$\mathrm{Al}$ volver a asumir Luis Castañeda como alcalde de la $\mathrm{Mu}$ nicipalidad de Lima en enero de 2015, comenzó una nueva etapa marcada por el conflicto abierto entre la comunidad shipiba y la Municipalidad de Lima Metropolitana. En un inicio, las nuevas autoridades municipales quisieron desconocer los acuerdos a los que se había llegado en el proceso de diálogo de años anteriores a los que se sumaba la situación de vulnerabilidad en la que se encuentra la comunidad desde el inicio de las obras de construcción. Esta situación comenzó a agravarse en el mes de enero, cuando se rompieron las tuberías de agua y de desagüe de la comunidad, contaminando con aguas servidas las calles y casas. Esta situación que pone en riesgo la vida y la salud de la comunidad fue también denunciada por la Defensoría del Pueblo en un oficio dirigi-

22 Este terreno está ubicado en Av. Malecón Checa S/N, Urb. Campoy, distrito de San Juan de Lurigancho y cuenta con una extensión de 7,141 $\mathrm{m}^{2}$. Este terreno fue comprado el 7 de octubre de 2014 y fue inscrito en Registros Públicos (partida $N^{\circ} 12231774$ ) a nombre del Fideicomiso. Este fue el tercer terreno que se propuso. Los dos primeros, más cercanos al actual lugar donde se encuentra la comunidad, pero en la orilla opuesta del río Rimac, no pudieron ser asegurados por la Municipalidad de Lima Metropolitana. 
do al alcalde Luis Castañeda a inicios del año 2015 (Oficio $\left.\mathrm{N}^{\circ} 09-2015 / \mathrm{DP}\right) .^{23}$

Sin embargo, la gestión del alcalde Castañeda reitera su negativa a retomar la reubicación de la comunidad. Así, el 20 de febrero de 2015, la Municipalidad de Lima Metropolitana responde a la Defensoría del Pueblo señalando que este asunto no es de su incumbencia sino de INVERMET. Sin embargo, INVERMET es un Organismo Público Descentralizado (OPD) de la propia Municipalidad de Lima, que además es presidida por el propio alcalde (Urrutia 2015).

Poco después el alcalde Castañeda anunció que los fondos destinados al Proyecto Parque Rímac y Río Verde se habían destinado a la construcción de otras obras de infraestructura de transporte urbano en la ciudad. Esta situación generó entonces la movilización de la comunidad de Cantagallo que organizó marchas y protestas en distintos puntos de la ciudad y frente a la Municipalidad de Lima Metropolitana. Estas protestas, junto a la presión mediática, hicieron que la Municipalidad finalmente decida retomar el diálogo con la comunidad.

Sin embargo, no existía por parte de la Municipalidad de Lima la intención de retomar el proyecto de reubicación. El alcalde Castańeda ya había utilizado los fondos del fideicomiso para otras obras y su propuesta para la comunidad shipiba era que se quede donde estaba, aunque las condiciones de

23 Es importante señalar que esta no ha sido la primera intervención de la Defensoría del Pueblo. Ya el año 2014 se había enviado por lo menos un oficio a la alcaldesa Susana Villarán recomendando que se apresure el proceso de reubicación (Oficio No 544-2014/DP), y posteriormente envió un segundo oficio al alcalde Castañeda (Oficio No 217-2015/DP). 
vida y salud se habían agravado. La construcción de las vías no solo había recortado el área de la comunidad, aumentando el hacinamiento, sino que, además, las obras de construcción y el tráfico vehicular generaban polvo y contaminantes que afectan directamente a la comunidad. Asimismo, no se solucionaban los problemas de agua, desagüe y electricidad; la escuela bilingüe funcionaba en condiciones más precarias; y se perdieron espacios comunales de encuentro. En este escenario, la comunidad sufrió un terrible incendio que la destruyó casi por completo.

En la madrugada del 4 de noviembre de 2016 se desató un incendio en Cantagallo que dejó más de dos mil personas damnificadas y prácticamente destruyó todas las casas de la comunidad. Aparentemente, el incendio comenzó en una tienda y se propagó rápidamente debido al viento y al hecho de que la mayoría de las viviendas estaban construidas con planchas de madera. Este hecho causó la indignación de la población limeña en general, generando una importante reacción de solidaridad para poder reconstruir sus viviendas, pero al mismo tiempo puso en evidencia la precaria situación en la que vivían las familias shipibas, así como la indolencia y responsabilidad moral del alcalde Castañeda que se había negado a continuar con el proyecto de reubicación de la comunidad. Es más, la propuesta de Castañeda frente al incen-

172 dio fue sugerir que el banco Mi-Vivienda les preste dinero a las familias shipibas para que construyan sus nuevas casas o compren departamentos en otras partes de la ciudad. Por supuesto, esta propuesta resultaba absolutamente ridícula y ofensiva en la medida en que la mayoría de familias shipibas no son elegibles para un préstamo debido a sus bajos ingresos, sino que además supone desconocer su deseo explícito de continuar viviendo como una comunidad. 
El gobierno nacional también reaccionó frente a esta situación, y algunas semanas después de ocurrido el incendio, el entonces presidente de la República, Pedro Pablo Kuczynski, visitó a la comunidad y prometió resolver las demandas de las familias shipibas. Luego de afirmar que: "No se puede vivir así, que eso quede claro. Debemos asegurarnos de que en esta zona no haya inundaciones o incendios", indicó que la comunidad contaría con servicios básicos como agua y electricidad, además de títulos de propiedad. Finalmente, prometió que en un año el gobierno podría brindarles todo lo que había ofrecido.

Sin embargo, las cosas han marchado a un ritmo más lento y la comunidad de Cantagallo ha seguido enfrentando muchas dificultades. Hacia abril de 2017, los líderes de la comunidad de Cantagallo se encontraban muy preocupados por la lentitud del proceso de reconstrucción y por la inseguridad jurídica en la que se encontraban. El problema principal surgía del hecho de que el Ministerio de Vivienda solicitaba que las familias de Cantagallo cuenten con los debidos títulos de propiedad sobre el terreno para poder iniciar la reconstrucción de las casas, sin embargo, al no ser reconocidos por el Estado como comunidad, les estaban obligando a conseguir títulos de propiedad individual. Los líderes de las distintas organizaciones buscaban el consejo de abogados y expertos para poder encontrar una solución a este problema. Una posibilidad que barajaban era la de constituirse como Asociación de Vivienda, o alguna otra figura jurídica que les permitiera mantenerse como comunidad.

Después de varios meses de incertidumbre y de trámites administrativos, recién hacia fines de agosto de 2017 se comenzó a entregar a las 238 familias shipibas un bono de 500 soles 
para poder buscar un alojamiento provisional hasta finalizar los trabajos de remoción de desmonte, nivelación y habilitación de los lotes donde se reubicarían a fines de 2018.

En octubre de 2017, el presidente Kuczynski volvió a visitar a la comunidad de Cantagallo y declaró que no solo se iban a reconstruir las viviendas perdidas un año antes en el incendio, sino que se iba a construir allí una "ciudad modelo". En esta ocasión dio más detalles sobre el proyecto y anunció que allí se construiría el nuevo conjunto habitacional, ahora rebautizado como Conjunto Habitacional "Buen vivir -Jakonax Jati Jema”, y que contaría con el apoyo del propio gobierno a través del Ministerio de Vivienda, Construcción y Saneamiento. Sin embargo, a diferencia del proyecto de la alcaldesa Villarán, se trataría ahora solamente de pequeñas viviendas de $50 \mathrm{~m} 2$ en lotes unifamiliares, y los únicos espacios comunes previstos son la escuela bilingüe y un espacio para la venta de artesanías.

Hacia mediados de 2018, las familias shipibas siguen viviendo dispersas en distintos puntos de la ciudad. En algunos casos, han tratado de buscar viviendas temporales que se encuentran cercanas entre sí, como en el caso del llamado "Cantagallo chico" o "Cantagallo chiquito", ubicado en el barrio de Campoy. En esta zona se han ubicado varias fa174 milias que también han comenzado a pintar las paredes de sus casas con diseños kené o con motivos relacionados con la cultura shipibo-konibo como una forma de apropiación del espacio y de expresión de su identidad. 


\section{Conclusiones}

A diferencia de otros pueblos indígenas de la Amazonía peruana, la migración shipiba a las ciudades ha generado la creación de comunidades indígenas urbanas donde se reproducen distintos aspectos de la vida y la cultura shipiba rural. El caso de la comunidad de Cantagallo en Lima es una muestra de este proceso. Sin embargo, el proceso de creación de estas comunidades se enfrenta a un Estado que no las reconoce formalmente como comunidades indígenas, generando situaciones de vulnerabilidad y de enfrentamiento abierto entre la comunidad y las autoridades municipales.

La comunidad de Cantagallo, al igual que las otras comunidades de indígenas urbanos, tienen que luchar, no solamente por el reconocimiento de sus derechos como indígenas; es decir, por el acceso a servicios y políticas públicas adecuadas a sus tradiciones culturales, como, por ejemplo, a la educación intercultural bilingüe o a la salud intercultural; sino que también tienen que estar permanentemente movilizándose para que se les reconozca su derecho a vivir como indígenas en la ciudad.

En los últimos cinco años, la comunidad de Cantagallo se ha visto envuelta, además, en los vaivenes y cambios de política por parte de las autoridades de la ciudad y del país. En este tiempo han tenido que enfrentar las decisiones arbitrarias tomadas por un gobierno municipal como el de Luis Castańeda, que se ha movido entre la indolencia y falta de escucha a la abierta discriminación y afectación de sus derechos básicos. Pero incluso, las decisiones aparentemente más favorables de la alcaldesa Villarán o del presidente Kuczynski no han concluido en el reconocimiento pleno de su derecho a vivir como una comunidad. 
A mediados de 2018, las familias shipibas viven dispersas y han buscado mantener sus vínculos comunitarios, como lo muestra el caso de "Cantagallo Chico". Sin embargo, ni las estrategias de adaptación a estos nuevos lugares ni las promesas oficiales logran despejar las dudas e incertidumbres que la comunidad mantiene ante la actuación las autoridades encargadas del proceso de construcción del nuevo complejo habitacional en Cantagallo: ¿cumplirán con los plazos establecidos?; ¿podrán volver a ocupar el espacio prometido?; ¿hasta cuándo recibirán los bonos para la vivienda temporal? Estas son algunas de las muchas preguntas que las familias shipibas se hacen desde la precariedad de su situación y frente a un Estado que todavía no les reconoce plenamente su derecho a vivir como comunidad indígena en medio de la ciudad.

Recibido: 12 de agosto del 2018.

Aprobado: 19 de diciembre del 2018.

\section{Bibliografía}

Ala, Miguel Ángel

(2014) "Shipibas de Cantagallo Incrementan Venta de Artesanía”, Revista Impresión, junio, pp. 15-17.

Alexiades, Miguel y Daniela Peluso

(2015)

"Introduction: Indigenous Urbanization in Lowland South America", The Journal of Latin American and Caribbean Anthropology, 20 (1):1-12.

Ananco, Dina

(2015) "Los shipibos no se alimeñan, buscan shipibizar a la capital”. Publicado el 17 de abril de 2015. Disponible en: <https://lamula.pe/2015/04/17/los-shipibos-no-se-alimenan-buscan-shipibizar-a-la-capital/ camazonico/> 
Anzualdo, Jesús

(2015) "Cartografiando la historia: desarrollo demográfico de la comunidad de Cantagallo". Ponencia presentada en el XI Congreso Nacional de Geografía "Ing. Pablo Sánchez Zevallos”. Geografía, desarrollo y sostenibilidad territorial: enfoques, políticas y estrategias. Cajamarca, Perú, 5 al 7 de octubre de 2015, en: <https:// www.academia.edu/17624354/Cartografiando_la_ historia._Desarrollo_demogr\%C3\%A1fico_de_la_ Comunidad_de_Cantagallo>

Anzualdo, Jesús

(2016) Identidady Socialización Interpretativa en Comunidades Escolares. Los shipibo de la I.E 'Comunidad Shipiba' del Rio Rimac (Cantagallo-Lima) y los cocama de la I.E Miguel Acobino del Rio Urituyacu (Nueva Alianza-Loreto). Tesis de Licenciatura en Antropología, Universidad Nacional Mayor de San Marcos.

Arana, Sara Isabel

(2011) "Cantagallo: Shipibos urbanos y la difícil tarea de construir una vida transcultural." Construyendo Nuestra Interculturalidad , 7 (6):1-10.

Arista Zerga, Adriana

(2011) "Pobres y excluidos en la selva de cemento: Los nativos shipibos de Cantagallo en Lima Metropolitana". En: M. Di Virgilio (ed.) Pobreza urbana en América Latina y el Caribe. Buenos Aires: Consejo Latinoamericano de Ciencias Sociales-CLACSO, pp.317-345.

Barclay, Frederica y Fernando Santos Granero

(1980) La conformación de las comunidades Amuesha: la

legalización de un despojo territorial, Amazonía Peruana, 3 (5): 43-74.

Bariola, Nino

(2009) "Apuntes sobre identidad, uso del lenguaje y migración: El caso de los shipibos en Lima", Revista Argumentos, 3 (2), disponible en: <http:// www.revistargumentos.org.pe/index.php?fp_ 
verpub=true \&idpub=161\&fp_plantilla_seleccionada_temporal $=74>$

Bariola, Nino

(2014) Migration, Ethnic Economy and Precarious Citizenship among Urban Indigenous People. Tesis de Maestría, Universidad de Texas, Austin.

Bonilla, Daniel

(2011) "Indígenas urbanos y derechos culturales: los límites del multiculturalismo liberal”, Revista Direito GV, 7 (2): 569-604.

Castillo, Daniel

"Pintando en Shipibo": el arte de Cantagallo en Lima desde un contexto sociocultural. Los casos de Elena Valera, Roldán Pinedo y, los hermanos Guimer y Rusber García. Tesis de Maestría en Antropología Visual, Pontificia Universidad Católica del Perú.

Colán, María Esperanza

(2009) Acceso a los servicios de salud: significado cultural en madres de la Asociación de Shipibos Artesanos en Lima-Ashirel 2008. Tesis para optar el título profesional de Licenciada en Enfermería. Lima: Universidad Nacional Mayor de San Marcos.

Chirif, Alberto.

(1980) Colonización e invasión: el despojo institucionalizado, Amazonia Indigena, 1 (1): 15-24.

Chirif, Alberto y Pedro García Hierro

(2007) Marcando Territorio: Progresos y limitaciones de la titulación de territorios indígenas en la Amazonía. Copenhague: International Work Group for Indigenous Affairs (IWGIA).

Chirif, Alberto et al.

(1991) El indigena y su territorio son uno solo. Estrategias para la defensa de los pueblos y territorios indigenas en la Cuenca Amazónica. Lima: Coordinadora de Organizaciones Indígenas de la Cuenca Amazónica (COICA), OXFAM América. 
Del Cairo, Carlos y Esteban Rozo

(2006) "Políticas de identidad, ciudadanía intercultural y reivindicaciones territoriales indígenas en dos localidades amazónicas", Universitas Humanística, 61: 107-34.

Espinosa, Oscar

(2004) Indigenous Politics in the Peruvian Amazonia: An Anthropological and Historical Approach to Shipibo Political Organization. Ph.D. Dissertation, New School for Social Research.

Espinosa, Oscar

(2009) "Ciudad e identidad cultural: ¿Cómo se relacionan con lo urbano los indígenas amazónicos peruanos en el siglo XXI?, Bulletin de l'Institut Français d'Études Andines, 38 (1): 47-59.

Espinosa, Oscar

(2012) "To Be Shipibo Nowadays: The Shipibo-Konibo Youth Organizations as Strategy for Dealing with Cultural Change in the Peruvian Amazon Region", Journal of Latin American and Caribbean Studies, 17 (3): 451-471.

Espinosa, Oscar

(2016) "La política indígena en la ciudad: El caso de las comunidades urbanas Shipibo-Konibo". En: A. Surrallés, O. Espinosa \& D. Jabin (eds.) Apus, caciques y presidentes: Estado y politica indigena amazónica en los paises andinos. Lima: IWGIA/ PUCP / IFEA, pp.103-122.

Frigola, Núria

(2016) Manifestación de la identidad étnica en la construcción de la agencia de migrantes indigenas amazónicos en la ciudad de Lima. Tesis de Maestría en Desarrollo Humano, Pontificia Universidad Católica del Perú.

Guima, Tilsa

(2011) "Entre dos aguas: De cómo surgió Cantagallo. La comunidad shipiba más grande en la ciudad de Lima", Construyendo Nuestra Interculturalidad, 7 (6):1-8. 
Huaroc, Judith

(2009) La concordancia gramatical en los sintagmas nominal y verbal del castellano de los shipibo del distrito de Yarinacocha (Ucayali). Tesis de Licenciatura en Lingüística, Universidad Nacional Mayor de San Marcos.

Kennedy, Lauren

(2011) "La pobreza móvil de los migrantes shipibo-conibo: Una investigación de la influencia de la migración en la cosmovisión shipibo-conibo de Canta Gallo - Rímac, Lima”, Independent Study Project (ISP) Collection. Paper 1080. <http://digitalcollections.sit.edu/ isp_collection/1080>

Lazarte, Javier

(2011) "Shipibo urbano: Urbanismo de una cultura indígena en el centro de Lima en el siglo XXI", Construyendo Nuestra Interculturalidad, 7 (6): 1-17.

Ludescher, Monika

(1986) "Las sociedades indígenas de la Amazonía en el derecho peruano: la 'Comunidad Nativa': Institución jurídica y realidad social”, Law and Anthropology, 1: 131-176.

Moscoso, Luis Enrique

(2010) "Entender(se) a(en) Lima. Los estudiantes shipibokonibo de Ucayali y sus estrategias de aprendizaje y uso del castellano en Lima y en sus escuelas públicas", Ponencia en el Congreso Iberoamericano de Educación "Metas 2021". Buenos Aires, 13-15 septiembre de 2010.

Narby, Jeremy

Visions of Land: The Ashaninca on Resource Development in the Pichis Valley in the Peruvian Central Jungle. Ph.D. Dissertation, Stanford University.

Peñaranda, María Claudia

(2015) Indigenas urbanos en la ciudad: aproximación etnográfica al estudio de caso de los asháninka del asentamiento humano "Horacio Zevallos" - Ate-Vitarte. 
Tesis de Licenciatura en Antropología, Pontificia Universidad Católica del Perú, Facultad de Ciencias Sociales.

Pinedo, Danny

(2014) The Politics of Sociality: Social Networks and Indigenous Mobilitacion in Peruvian Amazonia. Ph.D. Dissertation in Anthropology, University of Florida.

Quispe, Arturo

(2011)

"Educación Bilingüe Shipibo / Castellano En Lima: I.E. Comunidad Shipiba de Cantagallo", Construyendo Nuestra Interculturalidad ,7 (6):1-8.

Rosengren, Dan

(1987) In the Eyes of the Beholder: Leadership and the Social Construction of Power and Dominance among the Matsigenka of the Peruvian Amazon. Göteborg: Göteborgs Etnografiska Museum.

Sánchez, Janet

(2015)

"'Seguimos Siendo Indígenas': Una investigación sobre el discurso de los derechos y necesidades de la Comunidad Shipiba de Cantagallo como pueblo indígena en el contexto urbano de Lima", Independent Study Project (ISP) Collection, Paper 2137. < http:// digitalcollections.sit.edu/isp_collection/2137>

Sánchez, Liliana et al.

(2018) "Linguistic attitudes toward Shipibo in Cantagallo:

Reshaping indigenous language and identity in an urban setting", International Journal of Bilingualism, pp.1-22, doi: 10.1177/1367006918762164

Sánchez, Luisa F.

(2012) De totumas y Estantillos: Procesos migratorios, dinámicas de pertenencia y de diferenciación entre la Gente de Centro (Amazonía colombiana). Tesis para el Doctorado en Sociología, Université Sorbonne Nouvelle - Paris 3.

Santos Granero, Fernando y Frederica Barclay

(1985) "Las comunidades nativas: un etnocidio ideológico", Amazonia Indigena, 5 (9): 3-4. 
Sarfaty, Sara

(2011) La experiencia de ser estudiante universitario e indigena amazónico: prácticas discriminatorias al interior de la Universidad Nacional Intercultural de la Amazonia (UNIA). Tesis de Licenciatura en Antropología. Lima: Pontifica Universidad Católica del Perú.

Strauch, L. et al.

(2015) "Mixed-use spaces and mixed social responses: Popular resistance to a megaproject in Central Lima, Peru", Habitat International, 45: 177-184.

Suárez, Ronald

(2013) Lima Shipibo, filme documental disponible en Internet: <https://www.youtube.com/ watch?v=Zzlmvh9nIf0>

Surrallés, Alexandre y Pedro García Hierro (eds.)

(2004) Tierra Adentro: Territorio indigena y percepción del entorno. Lima: International Work Group for Indigenous Affairs (IWGIA).

Tintaya, Meir Álvaro

(2017) Empoderamiento psicológico en la Comunidad ShipiboKonibo de Cantagallo y sus relaciones con el sentido de comunidad y el bienestar. Tesis de Licenciatura en Psicología Social, Pontificia Universidad Católica del Perú.

Urrutia, Isabel.

(2015) "La larga lucha de los Shipibo Konibo de Cantagallo", 12 de marzo de 2015. Disponible en Internet: <http://www.justiciaviva.org.pe/notihome/notihome01.php?noti=1573>

182 Vega, Ismael

(2014)

Buscando el Río: Identidad, transformaciones y estrategias de los migrantes indígenas amazónicos en Lima Metropolitana. Lima: Centro Amazónico de Antropología y Aplicación Práctica (CAAAP), Terra Nuova, Municipalidad de Lima Metropolitana.

Zavala, Virginia y Nino Bariola

(2007) "Discurso, género y etnicidad en una comunidad de Shipibos en Lima”, Debates en sociología, 32: 50-69. 
(2008)

"Enra kopiai, non kopiai: Gender, ethnicity and language use in a Shipibo community in Lima”. En: M. Nino-Murcio \& J. Rothman (Eds.) Bilingualism and Identity: Spanish at the Crossroads with Other Languages. Amsterdam: John Benjamins, pp. 151-174

\section{Documentos inéditos:}

Ashirel-V (Asociación de Artesanos Shipibos Residentes en LimaPro-Vivienda)

(2011) Proyecto: Centro Turístico Ecológico Vivencial "Shipibo-Konibo". Lima: ASHIREL. Ms.

Morán, Dulce

(2012) Informe de Trabajo de Campo: "Dinámicas de atención en salud desdde el punto de vista del indigena amazónico migrante. El caso de los shipibos en el Hospital de Tingo María. Lima: Pontificia Universidad Católica del Perú. Ms.

Quintana, Andrea del Pilar

(2012) La comunidad emprendedora de Shipibos en Cantagallo-Rimac. Lima: Universidad Científica del Sur. Ms.

Quispe, Jorge

(2014)

Informe de Campo: Bena Jema II (Tingo María). Lima: Pontificia Universidad Católica del Perú. Ms.

Taller de Pre-Consulta

(2012)

Borrador de Acta: Acta del Taller del proceso pre-consultivo entre la Municipalidad de Lima y la comunidad urbana del pueblo shipibo-conibo asentado en Cantagallo relativo al proyecto Vía Parque Rímac - Linea Amarilla SAC. Ms.

Zegarra, Juan Carlos

(2009) De la selva tropical a la selva de cemento: El caso de los migranes Shipibos asentados en la zona de Cantagallo. Informe de Trabajo de Campo. Lima: Pontificia Universidad Católica del Perú. Ms. 
